\title{
Evaluate the Effect of Herbal Extract Remedy for Treatment of Liver Cirrhosis in In-Vitro
}

\author{
Thanh Ha Tuan Nguyen ${ }^{1,2, \#}$, Ngan Nguyen Hoang ${ }^{1, \#}$, Xuan Thanh Nguyen ${ }^{1,2}$, Binh Nhu Do ${ }^{1,2}$, Son Trinh The ${ }^{1, *}$
}

Thanh Ha Tuan Nguyen ${ }^{1,2, \#, ~ N g a n ~}$ Nguyen Hoang ${ }^{1, \#}$, Xuan Thanh Nguyen ${ }^{1,2}$, Binh Nhu Do ${ }^{1,2}$, Son Trinh The ${ }^{1, *}$

'Vietnam Military Medical University, No.160 Phung Hung st, Phuc La, Ha dong, Ha noi, VIETNAM.

${ }^{2}$ Military Hospital 103, No.261 Phung Hung st, Phuc La, Ha Dong, Ha Noi, VIETNAM.

"These authors contributed equally to this work and are co-first authors

\section{Correspondence}

\section{Associate Prof. Son Trinh The}

Vietnam Military Medical University, No.160 Phung Hung st, Phuc La, Ha dong, Ha noi, VIETNAM.

E-mail : trinhtheson@vmmu.edu.vn

History

- Submission Date: 06-10-2020;

- Review completed: 29-10-2020;

- Accepted Date: 09-11-2020.

DOI : 10.5530/pj.2021.13.27

Article Available online http://www.phcogj.com/v13/i1

\section{Copyright}

(C) 2021 Phcogj.Com. This is an openaccess article distributed under the terms of the Creative Commons Attribution 4.0 International license.

\begin{abstract}
Objectives: To evaluate the in-vitro effect of herbal extract recepies, namely XGTQ, in the treatment of liver cirrhosis that induced by Carbon tetrachloride (CCL4) in combination with alcohol and high-fat diet in rats. Materials and Methods: Liver cirrhosis was induced by subcutaneously injecting CC14 (initial dose of $5,0 \mathrm{ml} / \mathrm{kg}$, followed by $1,2 \mathrm{ml} / \mathrm{kg}$ twice a week in 10 weeks) in winstar rats. Then, fed with synthetic food, added $20 \%$ fat, and $0.05 \%$ cholesterol and iron oxalate. Rats were administered a day with fresh water and water mixed with $30 \%$ ethanol in another day. The rats were randomly divided into 5 groups and given distilled water (group 1 or control group and group 2 or cirrhosis group), silymarin (group 3 or reference group) or the herbal recipes, aka XGTQ, drug extract (group 4, 5) for 4 weeks. Blood was collected for biochemical test and livers were dissected to evaluate weight, morphology and quantified 4-hydroxyproline to evaluate fibrosis and collagen accumulation. Results: In cirrhotic wistar rats, the XGTQ herbal drug at $19.6 \mathrm{~g} / \mathrm{kg} / 24 \mathrm{~h}$ and $58.8 \mathrm{~g} / \mathrm{kg} / 24 \mathrm{~h}$ showed the ability of reducing the level of enzymes AST, ALT in the blood $(p<0.01)$, increasing plasma albumin and decreasing prothrobin time $(p<0.05)$; improving physical condition, macroscopic and microscopic images of H\&E-stained liver; decreasing the concentration of hydroxyproline in the liver and reducing the level of cirrhosis on the masson-stained templates. The effect of herbal recipes XGTQ increased dramatically with the dose, and was equivalent to silymarin at the dose of $70 \mathrm{mg} / \mathrm{kg} / 24 \mathrm{~h}$. Conclusion: The aqueous extract of XGTQ herbal remedy has have a good effect in treatment of liver cirrhosis in in-vitro and to be equivalent to that of silymarin at the dose of $70 \mathrm{mg} / \mathrm{kg}$.
\end{abstract}

Key words: XGTQ herbal extract, Liver cirrhosis, Wistar rats, CCl4, Silymarin.

\section{INTRODUCTION}

Cirrhosis is a diffuse, prolonged damage process in the liver, manifested by inflammation, necrosis of hepatic parenchyma cells; increasing fibrosis of the connective tissue causes scarring of sclerosis and formation of intact liver cells regenerating particles that upset the normal structure leading to the formation of tumors in liver parenchyma, losing the function of liver. Cirrhosis is a cause of increasing morbidity and mortality in developed countries. Worldwide, cirrhosis is the $14^{\text {th }}$ leading cause of death ${ }^{1}$. In the United States, cirrhosis is the 9th leading cause of death and accounts for $1.2 \%$ of all deaths in the US ${ }^{2}$. If not treated in time, cirrhosis will leave many serious life-threatening complications such as esophageal varices, liver coma, cancer, ...2.

The treatment of cirrhosis includes good compliance with diet and rest; treatment of symptoms such as improvement of metabolism, protection of liver cells (vitamins B, C, silymarin ..), albumin infusion when plasma albumin reduces, platelet transfusion in case coagulation disorders occurs...; treatment of complications (gastrointestinal bleeding, ascites, hepatorenal syndrome ...); and treatment depending the causes of cirrhosis.

Traditional medicine has many good remedies, most of them have a preventive and supporting effect in the treatment of cirrhosis, which slows down and reduces the process of cirrhosis. The "XGTQ" remedy, which has been used in the Department of Traditional Medicine - Military Hospital 103 to treat cirrhosis, was formulated from 12 medicinal herbs include Radix Astragali, Radix Codonopsis, Radix Glycyrrhizae, Rhizoma Atractylodis Macrocephalae, Radix Angelicae Sinensis, Pericarpium Citri Reticulatae, Radix Bupleuri, Frutus Schisandrae Chinensis, Poria, Zhizoma Alismatis, Concha Ostreae, Radix Curcumae. There have been many empirical and clinical studies to prove the effects of this herbs such as Frutus Schisandrae Chinensis, Radix Astragal, Radix Glycyrrhizae, Radix Bupleuri, Radix Curcumae...that worked well in treating cirrhosis.

This study was conducted to evaluate the therapeutic effect of the "XGTQ" extract drug in treatment of cirrhosis induced by CCL4 combined with alcohol and a high-fat diet in white rats.

\section{MATERIALS AND METHODS}

\section{Research drugs}

The herbal recipes includes the combination of 12 medicinal herbs, such as Radix Astragali 10g, Radix Codonopsis 10g, Radix Glycyrrhizae 10g, Rhizoma Atractylodis Macrocephalae 15g, Radix Angelicae Sinensis 10g, Pericarpium Citri Reticulatae 05g, Radix Bupleuri 05g, Frutus Schisandrae Chinensis 10g, Poria

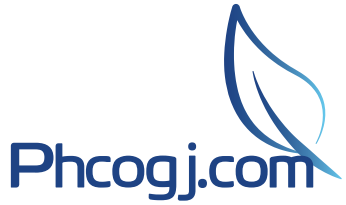

Cite this article: Nguyen THT, Hoang NN, Nguyen XT, DO BN, The ST. Evaluate the Effect of Herbal Extract Remedy for Treatment of Liver Cirrhosis in In-Vitro. Pharmacog J. 2021;13(1): 189-95. 
15g, Zhizoma Alismatis 15g, Concha Ostreae 20g, Radix Curcumae 15g. The herbs are provided by Mediplantex Central Pharmaceutical Company, that meet the requirement of Vietnam Pharmacopoeia V standards, extracted by hot water, packaged into $140 \mathrm{ml}$ high liquid 1:1 (01g of medicinal herbs $/ 1 \mathrm{ml}$ ) at the Department of Traditional Medicine - Military Hospital 103 using Korea's automatic dispensing and packaging system. The extract is concentrated using a vacuum evaporator to a ratio suitable for feeding to rats.

Reference drug

Silymarin (Milk Thistle component, HPLC $\geq 95 \%$, CAS Number 3388969-9, Sigma-Aldrich).

\section{Experimental animals}

Adult Wistar rats, irrespective of breed, 50 rats, met the required standards, weighed 160 - 180g, provided by Experimental Animal Husbandry Committee - Vietnam Military Medical University, raised in laboratory animals for at least one week before the experiment. Experimental animals were raised in an air-conditioned room at 22 $25^{\circ} \mathrm{C}$ with a $12 \mathrm{~h}$ light/dark cycle. All animals received a good care by well-training staffs, and the study protocols were performed according to the ethical guidelines for care and use of experimental animals approved by the Research Ethics Committee of Vietnam Military Medical University.

\section{Induction of liver cirrhosis}

The vistar rats were randomly divided into 05 groups (10 rats per group) as followed: group 1 were injected olive oil, rats in group 2, 3, 4 and 5 were induced cirrhosis by CCL4 in combination with alcohol and a high-fat diet $^{3-6}$. Briefly, conducted subcutaneous injection $\mathrm{CCl} 4$ mixed with $10 \%$ olive oil at the first dose of $5.0 \mathrm{ml} / \mathrm{kg}$. Then, injected twice a week CCL4 $1.2 \mathrm{ml} / \mathrm{kg}$ (on Monday and Thursday), injected for the next 10 weeks. At the same time, rats were fed with synthetic food, added $20 \%$ fat, $0.05 \%$ cholesterol and iron oxalate; administered alternately one day distilled water and one day water with $30 \%$ ethanol $70 \%$. Followed the above diet and injected CCl4 for 10 weeks to induce cirrhosis.

After the end of 10 weeks of inducing fibrosis, the rats were in group 1 administered distilled water and served as normal control. Group 2 administered distilled water and served as cirrhosis control group. Group 3 administered Silymarin $(70 \mathrm{mg} / \mathrm{kg} / 24$ hours, orally) and served as Silymarin group. Group 4 administered XGTQ (19.6 g/kg/24 hours, orally) and served as XGTQ dose 1 group. Group 5 administered XGTQ $(58.8 \mathrm{~g} / \mathrm{kg} / 24$ hours, orally) and served as XGTQ dose 2 group. Rats were forced to drink with curved head needle every day at 8 am, for 4 weeks. Assess the condition of study rats: weight, activity, fur condition. After the last 2 days of taking the drug, blood was taken to measure AST and ALT activity, plasma albumin, prothrombin time. Liver were dissected to evaluate morphology and weight.

\section{Histological examination}

Part of the mouse's liver (100g per mouse weight) was removed and fixed in $10 \%$ formalin for 24 hours. Then it was washed under tap water, dehydrated in ethanol $(50,70,80,90$, and $100 \%)$, cleared in xylene, and embedded in paraffin wax (with melting point at $55-60^{\circ} \mathrm{C}$ ). Liver sections ( $4 \mu \mathrm{m}$ thickness) were stained with $\mathrm{HE}$ and the Masson's Trichrome to evaluate the microscopic structure of the liver and the degree of cirrhosis. All the procedures were conducted at Department of Histopathology, Military Hospital 103.

The HE protocol included deparaffinization, alcohol passes, staining in Haematoxylin for $5 \mathrm{~min}$, chlorhydric alcohol $0.5 \%$ and ammonium hydroxide 1.5\% washes and staining in Eosin 1.5\% for $5 \mathrm{~min}$.
Other parts of the liver were stained with Masson's trichrome that was performed manually and the protocol included Harris' Haematoxylin, Briebrich Scarlet/acid fuchsin and Aniline Blue as reagents. For Picrosirius red staining, sections were stained in 0.1\% Direct Red 80/ Sirius Red F3B (C.I. 35780, Sigma-Aldrich, St. Louis, MO, USA) in saturated picric acid for $1 \mathrm{~h}$ at room temperature, and then the sections were differentiated in $0.5 \%$ acetic acid, prior to their dehydration, clearing and mounting.

Liver stained sections were examined using a light microscope (Olympus BX50, Japan) under high-power magnification $(\times 400)$. Fibrosis staging was defined according to International Association for Study of the Liver (IASL) as follows: stage 0: no fibrosis; stage 1: Mild fibrosis; stage 2: Moderate fibrosis; stage 3: Severe fibrosis; and stage 4: cirrhosis ${ }^{7}$.

\section{Hydroxyproline quantification}

Quantify the concentration of hydroxyproline in the liver using the method was described by Santh Rani Thaakur et al. ${ }^{4}$ to indirectly assess the amount of collagen in the liver (collagen in the hydrolyzed liver releases hydroxyproline). In brieft, took $500 \mathrm{mg}$ of liver, crushed homogeneously in $10 \mathrm{ml}$ of sulfosalicylic acid hydrated $3 \%$ water. Homogeneous liquid was filtered through Whatmann No. 2 filter paper. Took $2 \mathrm{ml}$ of filtrate (or standard material) into a test tube, added $2 \mathrm{ml}$ of ninhydrin acid and boiled for 1 hour. Added $4 \mathrm{ml}$ of toluene to the reaction mixture and stirred for 2 minutes. The toluene layering was separated, and warmed at room temperature. The intensity of the red substance was measured at $520 \mathrm{~nm}$. Compared with standard graph to calculate the concentration of hydroxyproline in liver.

\section{Ethical statements}

All procedures in animal experiment were approved by the Research Ethics Committee of Vietnam Military Medical University (Decision No. 29/2019/VMMU-IRB). All efforts were made to ameliorate harm to the animals, by conforming to the Principles of animal care and use in research adopted by the Vietnam Military Medical University

\section{Data analysis}

Data were processed using biomedical statistical methods, compared by ANOVA, post-test Turkey test, using SPSS 16.0 software. Data are presented in form of $\overline{\mathrm{X}} \pm \mathrm{SD}$. The difference was statistically significant when $p$ value $<0.05$.

\section{RESULTS}

\section{Physiological assessment in rats}

Rats with cirrhosis showed hairiness, hair loss, fatigue, reduction of activity, and weight loss compared to rats that did not induce cirrhosis. Rats in the XQTQ group significantly improved the above symptoms compared to the control group in the non-pharmacological cirrhosis group.

\section{Assessment of AST, ALT activity and hydroxyproline in rat liver}

The result in Table 1 showed that AST, ALT activity and hydroxyproline content in all cirrhosis groups increased significantly $(p-1<0.01)$ in comparison with the control group. The chemical agents $\mathrm{CCl} 4$, a diet rich in fat and alcohol increased the destruction of liver cells and damage of liver parenchyma. Those in the long run caused fibrosis of the liver, increase in the amount of collagen in the liver, thereby increased the content of hydroxyproline in rat liver.

For XGTQ extract at a dose of $19.6 \mathrm{~g} / \mathrm{kg} / 24$ hours and $58.8 \mathrm{~g} / \mathrm{kg} / 24$ hours showed the effect of reducing the level of liver parenchymal injury, decreased AST activity (47.09\%, 52.32\%), ALT activity (45.51\%, 
Table 1: Serum activity of AST, ALT activity and hydroxyproline content in rat liver $(\mathbf{n}=10)$.

\begin{tabular}{|c|c|c|c|c|c|c|}
\hline \multirow[t]{2}{*}{ Groups } & \multicolumn{2}{|c|}{ Serum activity of AST (U/L) } & \multicolumn{2}{|c|}{ Serum activity of ALT (U/L) } & \multicolumn{2}{|c|}{ Hydroxyproline content in the liver $(\mu \mathrm{g} / \mathrm{g})$} \\
\hline & $\bar{X} \pm S D$ & $\begin{array}{c}\text { \% decrease compared } \\
\text { to (2) }\end{array}$ & $\overline{\mathrm{X}} \pm \mathrm{SD}$ & $\begin{array}{c}\% \text { decrease com- } \\
\text { pared to ( } 2)\end{array}$ & $\bar{X} \pm S D$ & $\begin{array}{l}\% \text { decrease com- } \\
\text { pared to (2) }\end{array}$ \\
\hline Control group (1) & $116.39 \pm 18.55$ & - & $102.92 \pm 16.40$ & - & $47.61 \pm 7.79$ & \\
\hline Cirrhosis group (2) & $516.96 \pm 69.10$ & - & $494.88 \pm 57.42$ & - & $286.05 \pm 44.69$ & \\
\hline Silymarin (3) & $289.64 \pm 33,57$ & $43.97 \%$ & $279.12 \pm 33.00$ & $43.60 \%$ & $170.71 \pm 28.18$ & $40.43 \%$ \\
\hline XGTQ dose 1 (4) & $296.61 \pm 36.29$ & $42.62 \%$ & $281.48 \pm 25.98$ & $43.12 \%$ & $171.86 \pm 23.97$ & $39.92 \%$ \\
\hline XGTQ dose 2 (5) & $260.98 \pm 34.74$ & $49.52 \%$ & $250.74 \pm 33.67$ & $49.33 \%$ & $145.77 \pm 27.09$ & $49.04 \%$ \\
\hline $\mathrm{p}$ & & $\mathrm{p}_{2,3,4,5-1}<$ & $1 ; \mathrm{p}_{3,4,5-2}<0.01 ; \mathrm{p}$ & $.05 ; \mathrm{p}_{4-3}>0.05 ; 0,1>$ & $s_{5-3}>0.05$ & \\
\hline
\end{tabular}

$p_{23,45-1}-p$ value compared beetween groups $2,3,4,5$ to group $1 ; p_{3,5-2}$ - $p$ value compared beetween groups $3,4,5$ to group 2 .

$\mathrm{p}_{4-5^{\prime}}, \mathrm{p}_{4-3^{\prime}}, \mathrm{p}_{5-3}-\mathrm{p}$ value compared beetween group 4 to group 5, group 4 to group 3 , group 5 to group 3 , respectively.

$49.33 \%)$; reduced the level of cirrhosis by reducing hydroxyproline content in the liver $32.08 \%$ and $39.81 \%$ compared with the cirrhosis group. The difference was statistically significant with $\mathrm{p}<0.01$.

Comparison between 2 groups using XGTQ showed that in the highdose group, the activity of the enzymes AST, ALT and the concentration of hydroxyproline decreased compared with the low-dose group ( $\mathrm{p}$ $<0.05$ ), proving the effect of "XGTQ" increasing with dose. The activity of AST, ALT and hydroxyproline enzymes in the low-dose XGTQ group was not statistically different from the silymarin group ( $p>0.05)$, but the high-dose group tended to work better than in the silymarin group $(0.05<\mathrm{p}<0.1)$.

\section{Evaluation of plasma albumin, prothrombin time and liver weight}

In comparison to the control group, in cirrhosis group, plasma albumin concentration decreased, prothrombin time was longer, liver weight was statistically greater with $\mathrm{p}<0.01$. Inflammation and cirrhosis have increased the weight of the liver. A decrease in liver function caused a reduction in plasma albumin concentration and prolonged prothrombin time.

To compared with the cirrhosis group, XGTQ extract at dose of 19.6 $\mathrm{g} / \mathrm{kg} / 24$ hours and $58.8 \mathrm{~g} / \mathrm{kg} / 24$ hours showed the effect of reducing the level of cirrhosis at weight evaluation index, restoring liver function through increasing plasma albumin concentration and reducing prothrombin time. The difference was statistically significant with $\mathrm{p}$ $<0.05$.

The high-dose XGTQ group reduced liver weight, increased plasma albumin concentration and reduced prothrombin time better than in the low-dose XGTQ group and silymarin group through the evaluation of mean values, however, the difference was not statistically significant ( $>$ > 0.05) (Table 2).

\section{Evaluation macroscopic and microscopic images of rat liver}

Macroscopic features of rat liver: In the control group (Figure 1a), the liver surface was pink, smooth, liver density was soft. In the cirrhosis group (Figure 1b), the surface of liver was rough, there were many newly formed nodules, liver density was firm. The image showed noticeable improvement in the XGTQ dose 1 and XGTQ dose 2 groups (Figure 1d, 1e) or silymarin group (Figure 1c) with a smoother liver surface, newly formed nodules were not noticeable with less amount, liver density was softer.

Microsopic features of HE-stained rat liver: In the control group (Figure 2a), liver cells were normal with clear central veins, liver cords and hepatic lobules did not change in structure. In the cirrhosis group (figure $2 \mathrm{~b}$ ), the fibrous bands from the portal tracts developed and divided the liver lobules into false lobules. Fatty degenaration of liver cells occured and liver cell regeneration were formed. Bile ducts and capillaries proliferated at the portal tracts. The images showed noticeable improvement in XGTQ groups (figure $2 \mathrm{~d}, 2 \mathrm{e}$ ) or silymarin group (figure 2c), in which fibrous bands was thin, not noticeable, did not cause splitting of hepatic lobules to form false lobules, and the predominant images were fat degeneration of hepatocytes.

croscopic features of Masson-stained rat liver: The livers of the control group were not stained with Masson because they were normal. In the cirrhosis group (figure 3a), the fibrous bands in the portal tracts separating the liver lobes became clear green. The image showed noticeable improvement in XGTQ dose 1 and XGTQ dose 2 groups (figure 3c, 3d) or silymarin group (figure 3c), thin strips, slight discoloration (figures $3 \mathrm{~b}, 3 \mathrm{c}$ ), or unknown pronounced (figure $3 \mathrm{~d}$ ).

\section{DISCUSSION}

$\mathrm{CCl} 4$, alcohol when using alone or combine with a high-fat diet and/ or iron oxalate in a long time could induce fibrosis and cirrhosis ${ }^{3},{ }^{4}, 5$, ${ }^{6}$. However, the period of model was too long (16 weeks) ${ }^{6}$, or only the fibrosis appeared in liver, the cirrhosis was not clear ${ }^{3},{ }^{4}, 5$. In this study, $\mathrm{CCl} 4$, alcohol, high-fat diet and iron oxalate were used in combination during 10 weeks. The results showed that the cirrhosis was clear. In the cirrhosis group, AST, ALT activity and liver weight increased significantly $(\mathrm{p}<0.01)$ compared to the control group, indicating inflammation and damage to liver cells. This was an inevitable result of liver toxicity because CCL4 was combined with alcohol, a high-fat diet and iron oxalate. The process of inducing hepatitis lasted for a long period of time (10 weeks) leading to fibrosis and cirrhosis. Fibrosis of the liver was evident by increasing the concentration of hydroxyproline in the liver. The increase in hydroxyproline level in this study was higher than in the research of Santh Rani Thaakur ${ }^{4}$, probably because our fibrosis-induced process was longer, the fiber was stronger, and it caused cirrhosis actually. The image of liver cell damage and cirrhosis was clearly shown by $\mathrm{H} \& \mathrm{E}$ and Mason staining. Prolonged liver damage caused cirrhosis resulting in decreased liver function, decreased plasma albumin and prolonged prothrombin time $(\mathrm{p}<0.01$ compared to control group).

XGTQ herbal extract markedly improved liver cell damage and cirrhosis in cirrhosis-induced rats. Some of the indicators improving compared to the control group were plasma albumin content, prothrombin time and liver weight. Some other indicators did not recovered to the same level compared to the control group, including AST activity, blood ALT and hydroxyproline content in the liver, but the improvement compared to the cirrhosis group was significant $(p<0.01)$. Histopathological images also showed improvement of liver cell damage and cirrhosis.

Many herbs in XGTQ have been shown to work well in the treatment of cirrhosis. Schisandra reduces transaminase enzyme in patients with active chronic hepatitis ${ }^{8}$, preventing alcohol-related fatty liver disease in white rats ${ }^{9}{ }^{10}$. The whole extract as well as the active ingredient 
Table 2: Plasma albumin concentration, prothrombin time and liver weight in rats $(n=10)$.

\begin{tabular}{|c|c|c|c|c|c|c|}
\hline \multirow{2}{*}{ Groups } & \multicolumn{2}{|c|}{ Plasma albumin concentration (g/L) } & \multicolumn{2}{|c|}{ Prothrombin time (s) } & \multicolumn{2}{|c|}{$\begin{array}{l}\text { Liver weight } \\
(\mathrm{mg} / 100 \mathrm{~g})\end{array}$} \\
\hline & $\overline{\mathrm{X}} \pm \mathrm{SD}$ & $\begin{array}{c}\text { \% increase compared } \\
\text { to (2) }\end{array}$ & $\overline{\mathrm{X}} \pm \mathrm{SD}$ & $\begin{array}{c}\text { decrease compared } \\
\text { to (2) }\end{array}$ & $\bar{X} \pm S D$ & $\begin{array}{c}\text { \% decrease compared } \\
\text { to ( } 2)\end{array}$ \\
\hline Control group (1) & $31.93 \pm 1.76$ & - & $7.74 \pm 1.03$ & - & $2.51 \pm 0.27$ & \\
\hline Cirrhosis group (2) & $28.91 \pm 2.63$ & - & $9.28 \pm 1.09$ & - & $3.01 \pm 0.32$ & \\
\hline Silymarin (3) & $31.01 \pm 1.32$ & $7.24 \%$ & $8.33 \pm 0.62$ & $10.28 \%$ & $2.65 \pm 0.29$ & $11.83 \%$ \\
\hline XGTQ dose 1 (4) & $31.09 \pm 1.76$ & $7.53 \%$ & $8.35 \pm 0.65$ & $10.08 \%$ & $2.67 \pm 0.25$ & $11.23 \%$ \\
\hline XGTQ dose 2 (5) & $31.49 \pm 1.92$ & $8.90 \%$ & $8.09 \pm 0.75$ & $12.80 \%$ & $2.62 \pm 0.31$ & $13.09 \%$ \\
\hline $\mathrm{p}$ & & & $01 ; \mathrm{p}_{3,4.5-1}>0$ & $3.45-2<0.05 ; \mathrm{p}_{3,4-5}>0.05$ & 0.05 & \\
\hline
\end{tabular}

$p_{3,4,5-1}$ - $p$ value compared beetween groups $3,4,5$ to group $1 ; p_{3,4,5-2}$ - $p$ value compared beetween groups $3,4,5$ to group 2 .

$p_{2-1}, p_{3,4-55^{\prime}}, p_{4-3}-p$ value compared beetween group 2 to group 1 , groups 3,4 to group 5 , group 4 to group 3 , respectively.
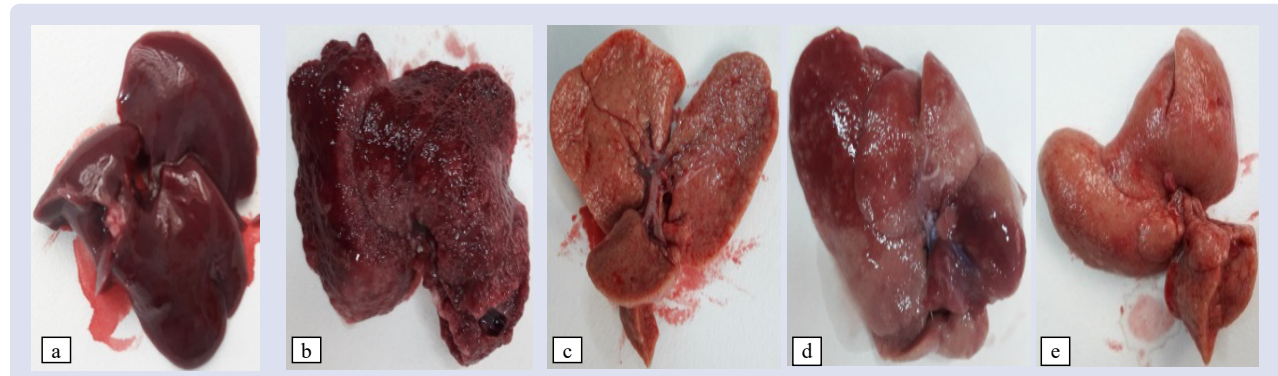

Figure 1: Macroscopic images of rat liver. a. control group; b. cirrhosis Group; c. Silymarin 70g/kg/24h group d. XGTQ $19.6 \mathrm{~g} / \mathrm{kg} / 24 \mathrm{~h}$ group; e. XGTQ $58.8 \mathrm{~g} / \mathrm{kg} / 24 \mathrm{~h}$ group.

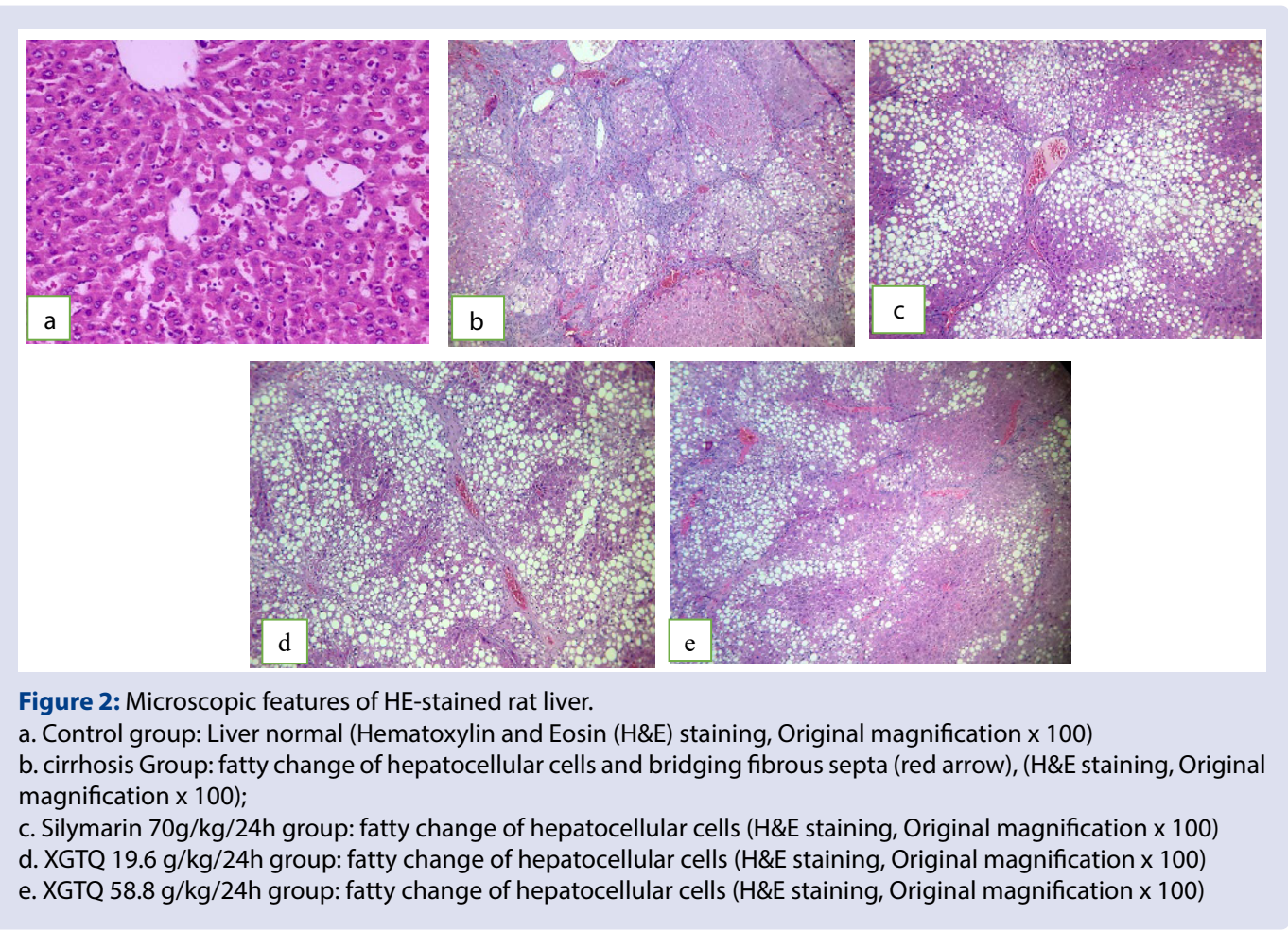




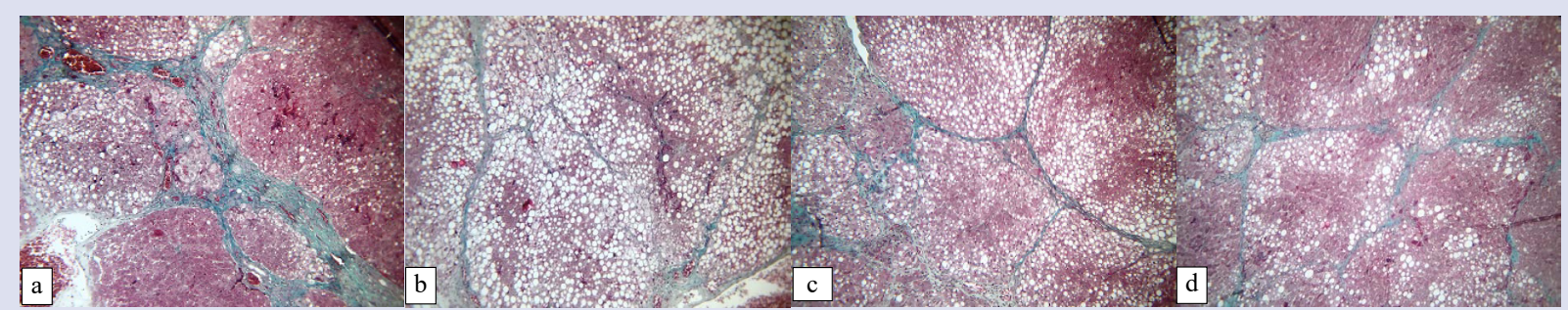

Figure 3: Microscopic features of Masson-stained rat liver.

a. cirrhosis group: blue bridging fibrous septa (Masson's Trichrome Staining Original magnification $x$ 100)

b. group of silymarin $70 \mathrm{~g} / \mathrm{kg} / 24 \mathrm{~h}$ : blue thin fibre in portal (Masson's Trichrome Staining Original magnification x 100)

c. group of XGTQ $19.6 \mathrm{~g} / \mathrm{kg} / 24 \mathrm{~h}$ : blue thin fibre in portal (Masson's Trichrome Staining Original magnification $\times 100$ )

d. group of XGTQ $58.8 \mathrm{~g} / \mathrm{kg} / 24 \mathrm{~h}$ : blue thin fibre in portal (Masson's Trichrome Staining Original magnification $\times 100$ )

Astragaloside IV extracted from Radix Astragali are effective against hepatitis B virus ${ }^{11}$. Total flavonoids of Radix Astragali showed antifibrotic effect on dimethylnitrosamine-induced liver cirrhosis in rats ${ }^{12}$. Licorice water extract has antioxidant effects, protecting liver cells in rats causing liver damage with $\mathrm{CCl} 4^{13}$. The total astragalus saponins, the main ingredient in Radix Astragali, when combined with glycyrrhizic acid, the main component in Licorice, has shown anti-cirrhosis effect both on invitro and invivo tests ${ }^{14}$. Radix Bupleuri's aqueous extract, given to rats for 3 days in advance, protects liver cells from acute damage by acetaminophen via CYP450 inhibitors ${ }^{15}$. Radix Curcumae has a good effect on capillary blood circulation ${ }^{16}$, is considered to play an important role in liver diseases especially cirrhosis. Radix Astragali has been reported to effectively treat chemical cirrhosis in rats but there have been no reports of individual use in treating cirrhotic patients. Some traditional remedies using Radix Astragali have been reported to work well in cirrhotic patients ${ }^{17,18}$. In patients with cirrhosis, Buzhong Yiqi Decoction significantly increased the level of serum magnesium, iron, zinc levels and significantly decreased the level of serum copper ${ }^{18}$, which shows the effectiveness in the treatment of cirrhosis. Because cirrhosis is a complex disease, a combination of many herbs with different effect targets is used to increase the effectiveness of treatment. Beside 7 herbs which are in Buzhong Yiqi Decoction (Radix Astragali, Radix Codonopsis, Radix Glycyrrhizae, Rhizoma Atractylodis Macrocephalae, Radix Angelicae Sinensis, Pericarpium Citri Reticulatae, Radix Bupleuri), the XGTQ remedy also has other herbs (Frutus Schisandrae Chinensis, Poria, Zhizoma Alismatis, Concha Ostreae, Radix Curcumae). All herbs of the XGTQ remedy are in combination according to the theory of traditional medicine in the treatment of cirrhosis. In this study, the effect of XGTQ remedy was evalutated on the cirrhosis induced by CCl4, alcohol, high-fat diet and iron oxalate. In rats with cirrhosis, cirrhosis on steatohepatic cells is very pronounced with manifestations of steatohepatic liver cells; splitting fibrous bands creating pseudo-lobules; liver hydroxyprolin and serum AST, ALT level were increased; plasma albumin was decreased; prothrombin time was prolonged ... All of these manifestations improved markedly when the rats were given oral XGTQ. The results of the treatment of cirrhosis in wistar rats of XGTQ extract initially confirmed the correctness in formulating the remedy to create the effect. These are the scientific bases for assessing the effectiveness of XGTQ remedy in clinic.

The effect of XGTQ extract increases in dose level. At high doses $(58.8 \mathrm{~g} /$ $\mathrm{kg} / 24 \mathrm{~h}$ ), the effect of reducing enzymes AST, ALT and hydroxyproline content was more significant than at low doses $(19.6 \mathrm{~g} / \mathrm{kg} / 24 \mathrm{~h})$.

In comparision to a comercial drug, Silymarrin, an active ingredient extracted from milk thistle which has been shown to be effective in the treatment of inflammation and cirrhosis, were used as a reference drug ${ }^{19}$. At a dose of $70 \mathrm{mg} / \mathrm{kg} / 24 \mathrm{~h}$, silymarin has the effect of reducing the activity of enzymes AST, ALT and the concentration of hydroxyproline equivalent to XGTQ extract dose of $19.6 \mathrm{~g} / \mathrm{kg} / 24 \mathrm{~h}$ ( $\mathrm{p}>0.05)$, tended to be inferior to the XGTQ extract dose of $58.8 \mathrm{~g} / \mathrm{kg} / 24 \mathrm{~h}(0.05<\mathrm{p}<0.1)$. The strong and weak effect is probably due to the dosage used, and further evaluation is needed.

\section{CONCLUSION}

Aqueous extract of herbal XGTQ remedy with doses of $19.6 \mathrm{~g} / \mathrm{kg} / 24 \mathrm{~h}$ and $58.8 \mathrm{~g} / \mathrm{kg} / 24 \mathrm{~h}$ has the effect of treating cirrhosis in the cirrhosisinduced rats model with CCL4 in combination with alcohol and highfat diet when assessed on the improvement of body condition, AST, ALT activity in blood, hydroxyproline content in liver, plasma albumin, prothrobin time, macroscopic features, microscopic features of the $\mathrm{H} \& \mathrm{E}$ and masson staining rat liver. The effects of "XGTQ" extract was increased in the dose level, and was equivalent to that of silymarin 70 $\mathrm{mg} / \mathrm{kg} / 24 \mathrm{~h}$.

\section{AUTHORS' CONTRIBUTION}

All authors contributed to data analysis, drafting or revising the article, gave final approval of the version to be published, and agree to be accountable for all aspects of the work.

\section{ACKNOWLEDGMENTS}

We thank all the staffs at the Laboratory of Experimental Pharmacology - Department of Pharmacology, Vietnam Military Medical University; Department of Histopathology - Military Hospital 103 for supporting the study.

\section{ETHICAL STATEMENTS}

All procedures in animal experiment were approved by the Research Ethics Committee of Vietnam Military Medical University (Decision No. 29/2019/VMMU-IRB). All efforts were made to ameliorate harm to the animals, by conforming to the Principles of animal care and use in research adopted by the Vietnam Military Medical University

\section{DECLARATION OF COMPETING INTEREST}

The author (s) declared no potential conflicts of interest with respect to the research, authorship, and/or publication of this article.

\section{DATA AVAILABILITY STATEMENT}

The data that support the findings of this study are available from the corresponding author, upon reasonable request.

\section{FUNDING}

The author(s) received no financial support for the research, authorship, and/or publication of this article. 


\section{REFERENCES}

1. Tsochatzis EA, Bosch J, Burroughs AK. Liver cirrhosis. The Lancet. 2014;383(9930):1749-61.

2. Suva MA. A Brief Review on Liver Cirrhosis: Epidemiology, Etiology, Pathophysiology, Symptoms, Diagnosis and Its Management. Inventi Rapid: Molecular Pharmacology. 2014.

3. Chheda TK, Shivakumar P, Sadasivan SK, et al. Fast food diet with CCl4 microdose induced hepatic-fibrosis-a novel animal model. BMC gastroenterology. 2014;14(1):89.

4. Thaakur SR, Saraswathy G, Maheswari E, et al. Inhibition of CCl 4-induced liver fibrosis by Trigonella foenum-graecum Linn. 2007.

5. Tsukamoto H, Towner SJ, Clofalo LM, French SW. Ethanol-induced liver fibrosis in rats fed high fat diet. Hepatology. 1986;6(5):814-22.

6. Tsukamoto H, Horne W, Kamimura S, et al. Experimental liver cirrhosis induced by alcohol and iron. The Journal of clinical investigation. 1995;96(1):620-30.

7. Desmet VJ, Gerber M, Hoofnagle JH, Manns M, Scheuer PJ. Classification of chronic hepatitis: diagnosis, grading and staging. Hepatology. 1994;19(6):1513-20.

8. Hop DC. Evaluating the effect of Schisandra chinensis (Turcz.) Baill. on reducing transaminase enzyme in patients with active chronic hepatitis, Hanoi Medical University; 1997.

9. Park HJ, Lee S-J, Song Y, et al. Schisandra chinensis prevents alcohol-induced fatty liver disease in rats. Journal of medicinal food. 2014;17(1):103-10.

10. Wang O, Cheng Q, Liu J, et al. Hepatoprotective effect of Schisandra chinensis (Turcz.) Baill. lignans and its formula with Rubus idaeus on chronic alcoholinduced liver injury in mice. Food \& function. 2014;5(11):3018-3025.
11. Wang $\mathrm{S}$, Li J, Huang $H$, et al. Anti-hepatitis B virus activities of astragaloside IV isolated from radix Astragali. Biological and Pharmaceutical Bulletin. 2009;32(1):132-5.

12. Cheng Y, Mai JY, Wang MF, Chen GF, Ping J. Antifibrotic effect of total flavonoids of Astmgali Radix on dimethylnitrosamine-induced liver cirrhosis in rats. Chin J Integr Med. 2017;23(1):48-54. doi:10.1007/s11655-016-2627-6.

13. Huo HZ, Wang B, Liang YK, Bao YY, Gu Y. Hepatoprotective and antioxidant effects of licorice extract against $\mathrm{CCl} 4$-induced oxidative damage in rats. International Journal of Molecular Sciences. 2011;12(10):6529-43.

14. Zhou $Y$, Tong $X$, Ren S. Synergistic anti-liver fibrosis actions of total astragalus saponins and glycyrrhizic acid via TGF- $\beta 1 /$ Smads signaling pathway modulation. Journal of ethnopharmacology. 2016;190:83-90.

15. Yu-Xin W, Yi D, Xia-Fei L. A hepatoprotection study of Radix Bupleuri on acetaminophen-induced liver injury based on CYP450 inhibition. Chinese journal of natural medicines. 2019;17(7):517-524.

16. Chen Z, Li W, Quan L. The Effects of Curcumae Longae Radix, Curcuma phaeocaulis Radix and Their Processed Products on Epo/EpoR Pathway and CD62p. Frontiers in pharmacology. 2018;9:736.

17. Feng, Y., Cheung, K., Wang, N. Chinese medicines as a resource for liver fibrosis treatment. Chin Med 4, 16 (2009). https://doi.org/10.1186/1749-8546-4-16.

18. Qian Weiwei, Chen Jingfan, Zhu Huiru. Study on Buzhong Yiqi Decoction to Trace Elements in Patients with Liver Cirrhosis. Guangdong Trace Elements Science. 2010-06

19. Federico A, Dallio M, Loguercio C. Silymarin/silybin and chronic liver disease: a marriage of many years. Molecules. 2017;22(2):191.

\section{GRAPHICAL ABSTRACT}

50 Wistar rats

(n= 10 each group)

$+\frac{1}{3}$

Rats were fed with synthetic food, added $20 \%$ fat, and $0.05 \%$ cholesterol and iron oxalate + were drank every day with fresh water, anotherday with water mixed with $30 \%$ ethanol.

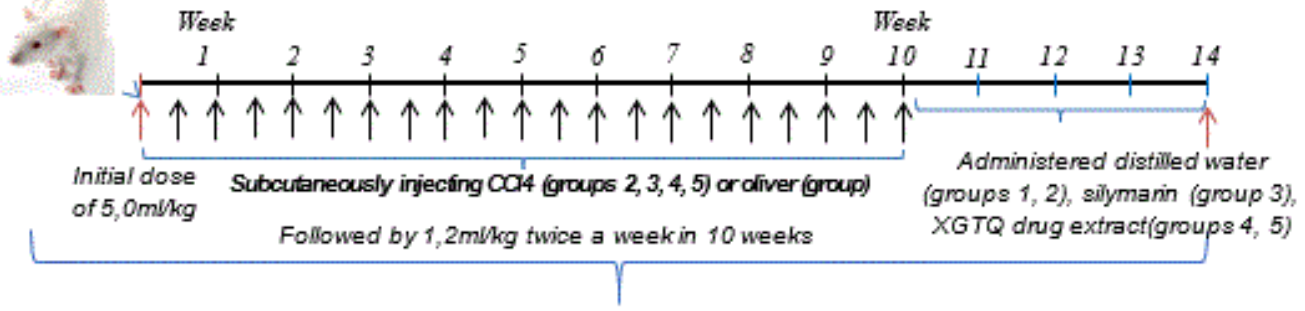

- Blood tests for AST, ALT activity, plasma albumin; Prothrombin time;

- Liver morphology, weight, H\&E staining, masson staining.

- Quantified 4-hydroxyprolin to assess fibrosis and collagen accumulation in the liver.

In groups 3, 4, 5 compared to in the cirrhosis group:

- Decressing AST, ALT activity ( $p<0.01$ );

- Increasing plasma albumin and decreasing prothrobin time $(p<0.05)$;

- Improving physical condition, macroscopic and microscopic images of H\&E-stained liver; decreasing the concentration of hydroxyprolin in the liver;

- Reducing the level of cirrhosis on the masson-stained templates.

$\downarrow$

The aqueous extract of XGTQ herbal remedy has have a good effect in treatment of liver cirrhosis in in-vitro, and was equivalent to silymarin at the dose of $70 \mathrm{mg} / \mathrm{kg}$ 


\section{ABOUT AUTHORS}
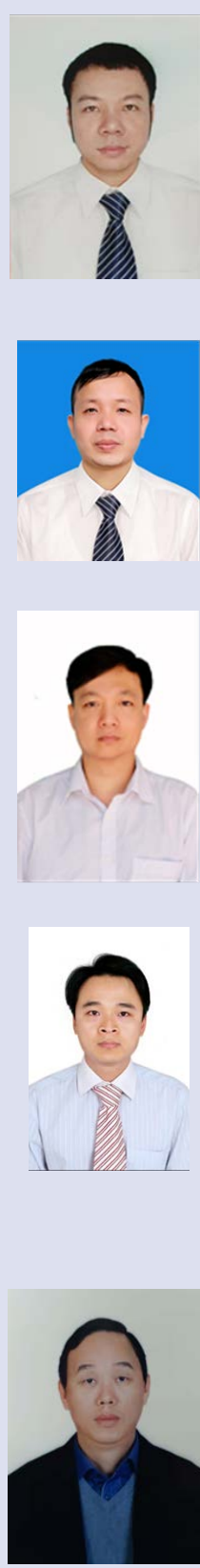

\section{Thanh Ha Tuan Nguyen}

Lecturer/Researcher

Deputy Head

Department of Traditional Medicine, Military Hospital 103, Vietnam Military Medical University

Email: nguyentuan000010@gmail.com

The main research fields: Research on medical remedies from natural herbs for application in scientific research and clinical treatment. Application of traditional medicine for treatment of patients at Military Hospital 103.

\section{Ngan Nguyen Hoang}

Lecturer/Researcher

Deputy Head

Department of Pharmacology, Vietnam Military Medical University, Phuc La, Ha Dong, Hanoi, Vietnam.

Email: nguyenhoangngan@vmmu.edu.vn

The main research fields: Biological, pharmacological and clinical effects of drugs and natural products

\section{Xuan Nguyen Thanh}

Doctor/Lecturer/Researcher

Department of Vascular Cardiology, Vietnam Military Medical University, Phuc La, Ha Dong, Hanoi, VietNam. Email: nguyenthanhxuan@vmmu.edu.vn

The main research fields: Methods of clinical diagnosis, subclinical, treatment of patients with cardiovascular diseases, musculoskeletal system, gastrointestinal tract, cancer and some other diseases.

\section{Binh Do Nhu}

Doctor/Lecturer/Researcher

Department of Infectious Disease, Vietnam Military Medical University.

Division of Military Science, Military Hospital 103, Ha Noi, Vietnam

Email: nhubinh.do@vmmu.edu.vn

The main research fields: My principal research of interest lies in the field of Infectious Diseases. I am currently investigating the impact of bacteria, viruses, parasites pathogens on human's health and using traditional and modern techniques to diagnose and cure the diseases caused by bacteria, viruses, parasites and others.

\section{Son Trinh The}

Doctor/Lecturer/Researcher

Academic title: Associate Professor

Vice Director of Military Institute of Clinical Embryology and Histology, Vietnam Military Medical University (VMMU)

Address: 222 Phung Hung road, Ha Dong district, Ha Noi, Vietnam

Email: trinhtheson@vmmu.edu.vn

The main research fields: Pathology and Molecular Pathology; Andrology; Histology; Endocrinology

Cite this article: Nguyen THT, Hoang NN, Nguyen XT, DO BN, The ST. Evaluate the Effect of Herbal Extract Remedy for Treatment of Liver Cirrhosis in In-Vitro. Pharmacog J. 2021;13(1): 189-95. 